\title{
Reexaminando el árbol de la teoría de la evaluación
}

\author{
Evaluation theory tree re-examined
}

\section{Christina A. Christie,, Marvin C. Alkin ${ }^{\text {II }}$}

I Profesora e investigadora de la Universidad de California (UCLA Graduate School \& Education and Information Studies) https://orcid.org/0000-00022842-5006

II Profesor emérito de la Universidad de California (UCLA Graduate School of Education \& Information Studies). https://orcid.org/0000-00022752-5777

Este artículo fue publicado en inglés, en 2008, en Studies in Educational Evaluation, 34, pp. 131-135. La traducción al español se publica con la debida autorización.

\section{Resumen}

Al examinar comparativamente varias teorías de evaluación, resulta útil tener un marco que muestre cómo se relacionan, destacando las características que distinguen a las diferentes perspectivas teóricas, en definitiva, una teoría acerca de las teorías. El árbol de la teoría de la evaluación que presentamos en un libro reciente de Alkin, Evaluation Roots (Las raíces de la evaluación) es un marco de este tipo [Eisner, E. The roots of connoisseurship and criticism: A personal journey; Guba, E., y Lincoln, Y. The roots of fourth generation evaluation: Theoretical and methodological origins; Stake, R. Stake and responsive evaluation; Wholey, J. S. Using evaluation to improve performance and support policy decision making. En M. Alkin (ed.) (2004). Evaluation roots: Tracing theorists' views and influences. Thousand Oaks, CA: Sage]. Así como los teóricos modifican sus puntos de vista a lo largo del tiempo, en este artículo sugerimos modificaciones al árbol teórico presentado en el libro, incluyendo un reposicionamiento de algunos teóricos, los aportes de nuevos teóricos y una reconceptualización en la rama de la valoración.

Palabras clave: evaluación, teoría de la evaluación.

\section{Abstract}

When examining various evaluation prescriptive theories comparatively, we find it helpful to have a framework showing how they are related that highlights features that distinguish theoretical perspectives, thus a 
«theory» about theories. The evaluation theory tree that we presented in Alkin's recent book, Evaluation Roots [Eisner, E. (2004). The roots of connoisseurship and criticism: A personal journey. In M. Alkin (Ed.), Evaluation roots: Tracing theorists' views and influences. Thousand Oaks, CA: Sage; Guba, E., \& Lincoln, Y., (2004). The roots of fourth generation evaluation: Theoretical and methodological origins. In M. Alkin (Ed.), Evaluation roots: Tracing theorists' views and influences. Thousand Oaks, CA: Sage; Stake, R. (2004). Stake and responsive evaluation. In M. Alkin (Ed.), Evaluation roots: Tracing theorists' views and influences. Thousand Oaks, CA: Sage; Wholey, J. S. (2004). Using evaluation to improve performance and support policy decision making. In M. Alkin (Ed.), Evaluation roots: Tracing theorists' views and influences. Thousand Oaks, CA: Sage], is such a framework. Just as theorists modify their views over time, in this paper we suggest modifications to the theory tree presented in the Roots book, including a repositioning of a few theorists, the addition of theorists, and a reconceptualization of the valuing branch. Keywords: evaluation, evaluation theorie.

\section{Introducción}

Cuando empezamos a pensar en este documento recordamos una cita de Egon Guba que Alkin había usado muchas veces. El escenario fue una reunión de la American Educational Research Association en la que un grupo de estudiantes de Alkin interpretaban el papel de diferentes teóricos en un juego de roles. Cada estudiante, en su rol, debía indicar cómo modificaría su punto de vista, basándose en las ideas presentadas por dos filósofos belgas. Guba, como comentarista del documento estudiantil que reflejaba sus opiniones, dijo:

Verás, en muchos sentidos yo no soy el «verdadero» Egon Guba —al menos no el que Lindheim tenía en mente. Cuando ella llevó a cabo su ejercicio, Egon Guba era el que trabajaba y escribía hace siete o diez años... mi pensamiento ha cambiado sobre tantas cosas que es difícil para mí recordar qué perspectiva tenía entonces. (1979, p. 139)

La declaración de Guba nos recuerda que los puntos de vista cambian con el tiempo. La gente lee cosas. Las ideas flotan. Las interpretaciones se modifican. Esa es una de las maneras en que las teorías sobre la evaluación se desarrollan y cambian con el tiempo. Pero, incluso considerando la posibilidad de que los cambios puedan tener lugar, es importante tener un marco conceptual explícito. Tales marcos de referencia ayudan a orientar a los profesionales a la hora de elegir el enfoque a utilizar, además de 
servir como valiosos instrumentos de enseñanza, porque ofrecen representaciones comparativas, descripciones relacionales de enfoques.

Al pensar en las diversas teorías de evaluación, resulta útil tener una teoría sobre cómo se relacionan entre sí: un marco de teorías. El árbol de la teoría de la evaluación que se plantea en el reciente libro de Alkin, Evaluation Roots (Raíces de la evaluación) (2004), es un sistema. Nuestra opinión es que hay tres elementos básicos cuando consideramos las teorías de la evaluación: utilización, métodos y valoración. Todos los teóricos se preocupan por los métodos que se emplearán para realizar una evaluación y reconocen que la evaluación es una empresa que implica valorar (distinguiéndose de la mayoría de las investigaciones). Todos los teóricos reconocen que las evaluaciones se utilizarán en formas que afectan a los programas. Postulamos, sin embargo, que los teóricos difieren en el énfasis particular que ponen en una u otra de estas dimensiones, a las que nos referimos como ramas del árbol de la teoría de la evaluación. Por tanto, podemos colocar a los teóricos en la rama que mejor refleja su énfasis principal de una manera que refleje alguna combinación de historia y/o la influencia de un enfoque particular en otro (ya sea sobre la base o en respuesta a los principios de un enfoque particular).

A medida que los teóricos modifican sus puntos de vista con el tiempo, proponemos modificaciones al árbol de la teoría de la evaluación (versión 3, figura 2), que reflejan algunos cambios sustanciales en nuestro pensamiento. En este artículo sugerimos modificaciones adicionales del árbol teórico presentado en el libro Evaluation Roots (Raíces de la evaluación) (véase figura 1). La figura 2 muestra el árbol tal como lo percibimos ahora.

\section{El árbol de la teoría de la evaluación de 2004: breve resumen}

Después de varios años de conversaciones en profundidad sobre la teoría y los teóricos de la evaluación, decidimos ilustrar la relación y mapear libremente la evolución del desarrollo de la teoría de la evaluación, centrándonos principalmente en el trabajo que surgió en América del Norte. Nuestro árbol de la teoría de la evaluación fue el producto de este esfuerzo. Debemos reconocer aquí, como lo hicimos en la versión original del árbol, que no estamos hablando de teorías tal como las definen los estándares académicos. Si bien el término teoría se usa convencionalmente en la literatura de evaluación, es más apropiado usar los términos enfoques, modelos o, incluso, marcos. Las teorías de evaluación son casi exclusivamente prescriptivas, es decir, ofrecen un conjunto de reglas, prescripciones y prohibiciones que especifican lo bueno o efectivo en 
un estudio de evaluación y cómo debe ser realizada una evaluación. Ninguno de los enfoques de evaluación es predictivo ni ofrece una teoría empírica. Sin embargo, en la literatura de evaluación se ha convenido llamar teorías a nuestros enfoques prescriptivos, por lo que también nos referimos a los modelos descritos en este artículo como teorías y a quienes han desarrollado estos modelos como teóricos.

El primer árbol de la teoría fue presentado y descrito con gran detalle en el capítulo 2 del libro Evaluation Roots, de Alkin (2004). En ese capítulo, Alkin y Christie discuten 27 enfoques de evaluación diferentes y clasifican a cada uno por su enfoque principal en uno de los tres elementos esenciales de evaluación, métodos, valoración y utilización. Los autores postulan que todas las teorías de la evaluación tienen muy en cuenta las cuestiones relacionadas con: 1) los métodos utilizados en una evaluación, incluido el diseño del estudio; 2) la manera en que los datos deben ser juzgados y valorados y por quién, y los valores subyacentes utilizados para lograr esto; y 3) la utilización del esfuerzo de evaluación. El capítulo final del libro ofrece un análisis del esquema de clasificación a la luz de la información proporcionada por los teóricos sobre su trabajo en el texto (Evaluation Theory Tree Revisited) (árbol de la teoría de la evaluación revisado), y se presentó una segunda versión del árbol de la teoría de la evaluación (véase figura 1), que difiere solo ligeramente de la presentada anteriormente.

En el último capítulo del libro Evaluation Roots ya reconocimos cambios de la encarnación original del árbol (presentado en el capítulo 2), estimulados por las anotaciones escritas por varios teóricos. Estos cambios fueron relativamente menores. En general, fueron simplemente reposicionamientos de las subramas de diferentes maneras para reflejar hasta qué punto un teórico de una rama particular tendía a las opiniones reflejadas en otra rama. Además, habíamos señalado dos fundamentos para el árbol de la teoría: la tradición de la investigación social y el deseo de responsabilidad y control. En respuesta a los comentarios de Yvonna Lincoln cambiamos uno de los fundamentos y pasó a llamarse rendición de cuentas a la sociedad y control fiscal (social accountability and fiscal control).

Nuestro árbol de la teoría de la evaluación consiste en un tronco y tres ramas. El tronco se apoyó inicialmente en las raíces fundacionales de la rendición de cuentas social y la investigación social sistemática. La rama media del árbol crece desde la raíz fundacional de la investigación social. El enfoque principal de los que se colocan en esta rama es desarrollar modelos para la práctica de la evaluación que en el fondo se basan en y se derivan de los métodos de investigación de las ciencias sociales. Los modelos teóricos son en su mayoría derivaciones del rastro del control aleatorio, y tienen como objetivo ofrecer resultados que sean generalizables y tengan un enfoque en la construcción del conocimiento. La rama de la valoración 1 se centra en aquellos teóricos que, como característica central de sus teorías de evaluación, consideran que el proceso de dar valor a la evaluación es el componente esencial del trabajo de un evaluador. Esto se 
extiende hasta incluir a teóricos que abogan por que el evaluador facilite sistemáticamente la determinación del valor por parte de otros. La tercera rama principal es la utilización. Los teóricos de la utilización están más preocupados por el uso de la evaluación y si se utiliza la información generada por la evaluación, y se centra en los que usarán la información.

Figura 1

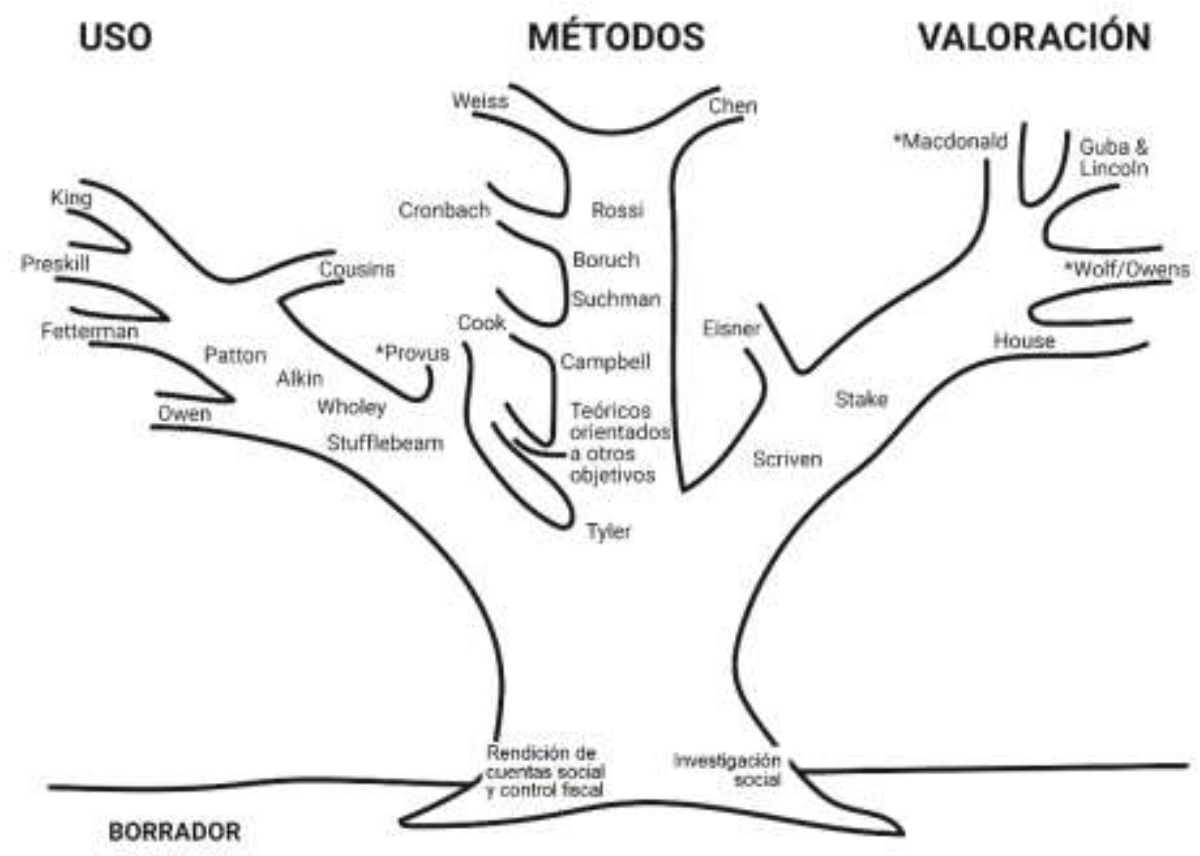

*No incluido en los capitulos

\section{El árbol de la teoría de la evaluación de 2006}

La figura 2 presenta la tercera versión de nuestro árbol de teoría de la evaluación. Las modificaciones de las ramas de utilización y métodos son relativamente menores, en particular, cuando se comparan con la revisión en la rama de la valoración. Los autores invitan a comentar y criticar las modificaciones presentadas, ya que consideramos que este trabajo está en curso, que requiere más reflexión y análisis. 


\section{Revisiones a la rama de la utilización}

En su más reciente encarnación, la rama de utilización se sometió sólo a una revisión menor y todavía se asemeja mucho al árbol presentado en el capítulo 26 del libro Evaluation Roots. Las ligeras modificaciones incluyen el reposicionamiento de la obra de Joseph Wholey (2004) para reflejar un énfasis hacia la rama de los métodos. Además, hemos eliminado a Malcolm Provus porque gran parte de su trabajo ha tenido relativamente poca influencia en otras perspectivas de esta rama.

\section{Revisiones a la rama de los métodos}

La rama de métodos recibió cambios más sustanciales. Ralph Tyler fue reposicionado en una subrama para reflejar que mientras su punto de vista teórico estaba, de hecho, muy relacionado con los métodos (evaluación basada en objetivos), no fue un predecesor teórico de los que están más arriba en la rama. Su posición original reflejaba su influencia en el campo de la evaluación educativa (que fue muy significativa), pero al continuar la reflexión llegamos a la conclusión de que su influencia general en la rama de métodos - específicamente - fue menor de lo que su posición original hacía suponer. Por lo tanto, lo colocamos en una pequeña subrama cerca de la base de la rama de métodos.

Donald Campbell sigue siendo el corazón y el alma de esta rama, tanto por la forma en que influyó directamente en otros teóricos con su trabajo en experimentos, cuasiexperimentos y validaciones como por la forma en que sus opiniones sirvieron de base para contrarrestar, particularmente, al respecto de las opiniones de Lee Cronbach.

También hemos eliminado a Edward Suchman de la rama. Reconocemos la importante influencia de la escritura de Suchman en la evaluación, particularmente en el posicionamiento de la obra de Campbell en un lugar prominente en el discurso de la evaluación. Sin embargo, a pesar de su importancia histórica, no parecía apropiado continuar incluyéndolo en la rama porque él mismo no ofreció al campo un modelo de evaluación específico. Más bien, promovió el trabajo de Campbell como el enfoque más eficaz para realizar evaluaciones para medir el impacto de un programa. Peter Rossi sigue apareciendo en la parte principal de la rama como una influencia en ambos: en Carol Weiss y, más particularmente, en Huey Chen.

Un cambio significativo en esta rama fue mover a Tom Cook que, previamente, había sido posicionado en una subrama que fluía desde Campbell. Ahora se encuentra en la rama principal, y su subrama fue eliminada. Se creó una nueva subrama para Bob Boruch. Esta viene directamente de Campbell y Cook y su ubicación refleja la firme influencia de los ensayos aleatorios controlados (RCT) de Campbell en el trabajo de 
Boruch. La rama se compensa con una subrama para enfatizar su salida de la rama de Cook y Campbell por su falta de consideración por los cuasiexperimentos. Esta posición también balancea la posición de Boruch respecto al resto de los teóricos de la rama de los métodos, todos los cuales han adoptado los cuasiexperimentos como un método aceptable para estudiar la causalidad, aunque todos en la rama estarían de acuerdo en que el experimento es ideal si el contexto y las condiciones lo permiten.

Otro cambio importante en esta rama es la adición de Gary Henry, Mel Mark y George Julnes. Como fue dicho en el libro de Evalution Roots:

Las teorías incluidas pudieron ser clasificadas en una sola rama del árbol... No está claro si algunas teorías no fueron incluidas debido a su amplitud o a nuestra incapacidad conceptual. Un ejemplo particular nos viene a la mente: el trabajo de Mark, Henry y Julnes (2000). Estos autores ven la mejora social como el objetivo final de la evaluación y presentan un punto de vista basado en lo que ellos llaman filosofía realista del sentido común... La naturaleza muy diversificada de esta perspectiva, aunque con una gran fuerza en la presentación de una comprensión de la evaluación, impide su inclusión en el árbol. (pp. 58 y 59)

Admitimos que en este caso nuestro análisis conceptual fue incompleto. Nuestras opiniones para determinar la exclusión se basaron en el libro de Mark et al. (2000). Sin embargo, al reflexionar más adelante sobre los escritos de estos autores, nos llamó la atención los puntos de vista presentados en la monografía de la evaluación realista en New Directions for Evaluation (Henry, Julnes y Mark, 1998). Basados en una lectura más profunda de este texto, hemos colocado a Henry, Mark y Julnes en la rama de la sección de métodos, inclinándose hacia la rama de valoración pospositivista. La inclinación destaca la colaboración de Henry y Mark con George Julnes para crear «una nueva teoría que capta las contribuciones de dar sentido del pospositivismo y la sensibilidad a los valores de las tradiciones constructivistas» (Henry et al., 1998, p. 1).

Henry, Mark y Julnes describen su enfoque, Emergent Realist Evaluation (Evaluación realista emergente), como un nuevo modelo de evaluación integral que ofrece nociones reconceptualizadas de utilización, métodos y valoración. La valoración y la utilización son aspectos importantes de la teoría de Mark y Henry, sin embargo, la metodología es el pilar fundamental. Este enfoque es: 1) una metodología de evaluación que da prioridad al estudio de los mecanismos generativos; 2) está atento a múltiples niveles de análisis; y 3) combina métodos apropiadamente. Los mecanismos se definen como «las causas subyacentes de los cambios que son observados» (Mathison, 2005, p. 360). Henry, Mark y Julnes también ven la evaluación como una herramienta para el cambio social y político dentro de las democracias. En sus palabras, «la mejora social, en lugar del más popular y omnipresente objetivo de utilización, debería motivar la evaluación» 
(Mark, Henry y Julnes, p. 19). Basados en esta descripción, colocamos a Henry, Mark y Julnes en la rama de métodos del árbol, en un lugar a continuación de Tom Cook, inclinándose hacia la rama de la valoración.

\section{Revisiones a la rama de la valoración}

La rama de la valoración ha recibido la revisión más sustancial. Esta rama siempre ha sido la más difícil de explicar en relación con su evolución. Es obvio que el trabajo de Scriven ha influido significativamente. Después de todo, es Scriven quien proclama que una evaluación no es una evaluación sin valoración; en sus palabras, la evaluación es la ciencia de la valoración (Scriven, 2003). El trabajo del evaluador es hacer un juicio de valor sobre el objeto que está siendo evaluado. Este pensamiento ha dado forma y ha definido el campo. Sin embargo, esta rama también incluye el trabajo de los interesados en la justicia social de la evaluación, así como a quienes defienden la filosofía de la subjetividad, es decir, la afirmación de que no hay una realidad objetiva. Aquí los teóricos también se preocupan por la valoración, pero se hace hincapié en cuáles valores dan forma a la evaluación. Hay preguntas sobre qué valores dan forma a la evaluación, en términos de por qué y con qué intención. Esto es muy diferente del tipo de valoración que concierne a Scriven. Con un examen cuidadoso ha quedado claro cómo estos dos focos difieren.

Con poca sorpresa, examinar las perspectivas teóricas incluidas en la rama de la valoración en el contexto de la filosofía de la ciencia ofrece una comprensión revisada de cómo entendemos estas perspectivas. Los axiomas básicos de los paradigmas pospositivista y constructivista ofrecen un marco más claro para categorizar los modelos de evaluación en la rama de la valoración. De modo que hemos elegido dividir la rama de la valoración en dos, nombrando al brazo izquierdo, que se extiende hacia la rama de los métodos como valoración: influencia pospositivista y al brazo derecho como valoración: influencia constructivista. Es importante destacar el uso de la palabra influencia en nuestra descripción de la nueva rama correspondiente a la valoración. Es decir, algunas perspectivas en la rama de la valoración están modeladas exactamente por un paradigma, mientras que otras solo sugieren una tendencia a un paradigma subyacente.

Las opiniones de la ciencia cambiaron, sin embargo, durante el siglo XX, desde el positivismo al pospositivismo. Los pospositivistas reconocen que toda observación es falible y tiene errores. Donde los positivistas creían que el objetivo de la ciencia era descubrir la verdad, el pospositivismo cree que el objetivo de la ciencia es intentar medir la verdad, aunque ese objetivo no pueda ser obtenido. El constructivismo es un elemento del interpretativismo (interpretivism) y, ontológicamente, adopta una postura relativista. No hay una única y tangible realidad que puede ser aproximada; solo hay múltiples 
realidades construidas. Epistemológicamente, el constructivismo ve a la subjetividad como la única realidad, es decir, la única forma en que lo desconocido puede llegar a ser conocido es a través de nuestro propio sistema de creencias.

Figura 2

uso

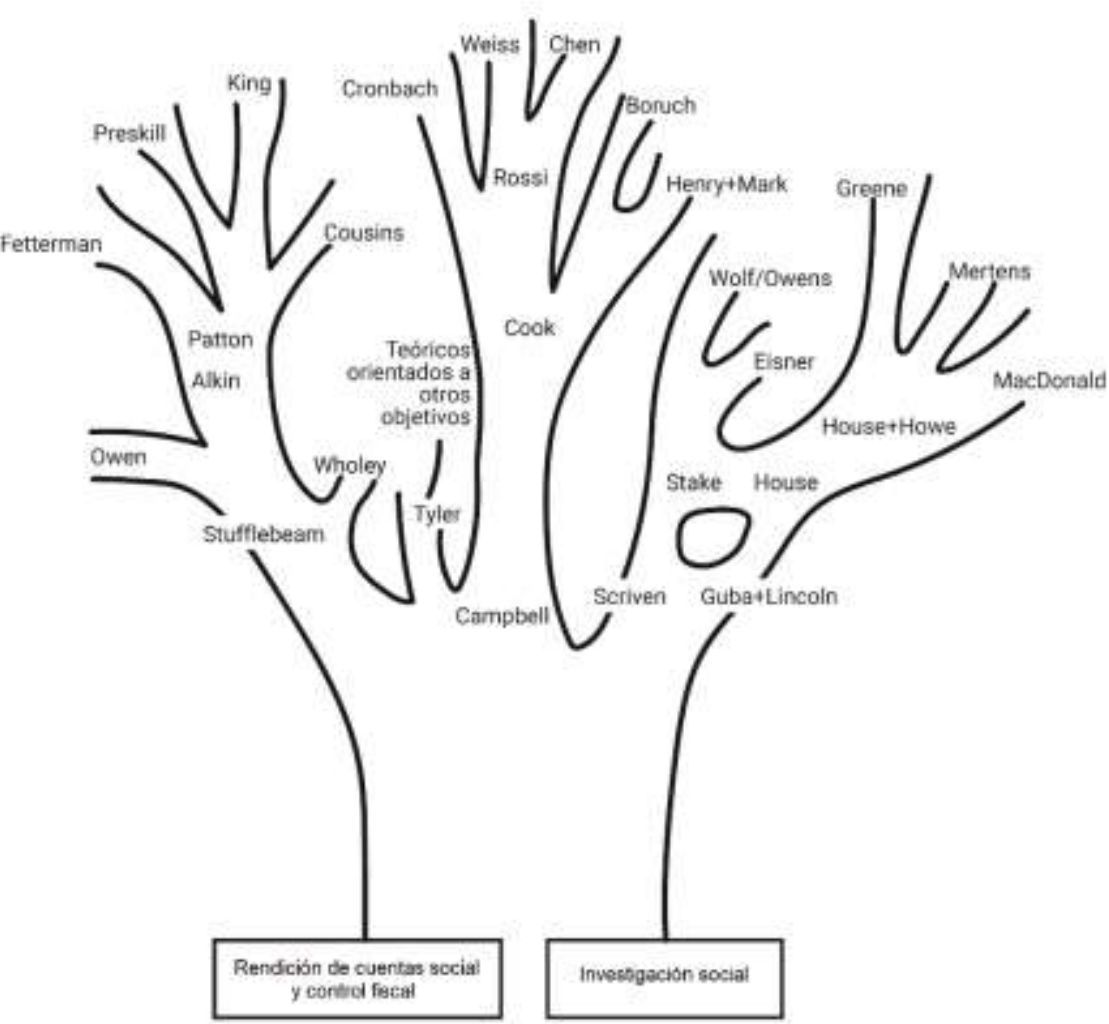

El pensamiento realista de Scriven sobre la valoración refleja tanto la ontología como la epistemología del paradigma pospositivista. No argumenta en contra de la idea de que deberíamos buscar una verdad objetiva sobre el objeto que se está evaluando. Él, de hecho, ofrece lo que cree que es un método comparativamente insesgado para obtener la verdad sobre el valor de un objeto, y luego aboga para que el evaluador haga un juicio de valor después de reunir la evidencia más creíble. Tampoco rechaza la idea de usar experimentos para determinar la causalidad, sino que argumenta que debería 
aplicarse más de un método para ello. (Donaldson, Christie y Mark, 2008). El pensamiento de Scriven, más que ningún otro, empujó al campo a considerar la valoración como una característica central de la evaluación. No obstante, ha sido reposicionado en la rama de la valoración, en la base del brazo de influencia pospositivista, para reflejar la ontología y la epistemología de su perspectiva.

La obra de Stake (2004) respeta el pensamiento de Scriven, aunque él aboga por el uso de la descripción gruesa para evaluar el valor de un programa a través del método del estudio de casos. El uso de los métodos de estudio de casos introdujo la idea de que el valor está ligado al contexto y que los evaluadores deben tener en cuenta el contexto al determinar el valor. Stake, sin embargo, deja la valoración al evaluador y, por lo tanto, no parece rechazar la idea realista de que la evaluación es una ciencia de la valoración. Fue la inclusión y el énfasis de Stake en el método de estudio de casos y el contexto del programa lo que provocó un pasaje desde el realismo (realist) hacia modelos de investigación relativista en la evaluación. Así, Stake se posiciona sobre un puente, directamente sobre la brecha de los dos énfasis de la rama de la valoración.

El trabajo de House (p. ej., House, 1999) atrajo una atención significativa a la inclusión de los valores de los subrepresentados al proceso de evaluación. Ontológica y epistemológicamente, el trabajo de House se basa en el pensamiento constructivista, aunque metodológicamente su trabajo rompe con los axiomas básicos del paradigma constructivista. Es decir, es proclive al uso de métodos cuantitativos para realizar las evaluaciones. Así, él también se sitúa sobre el puente, a la derecha de Stake. El trabajo de Eisner (2004) es ahora representado como un retoño de la rama de Scriven hacia el brazo de influencia constructivista. Eisner es similar a Scriven en tanto plantea que el evaluador es el experto y, por lo tanto, determina el valor final de un programa. Se diferencia de Scriven en el sentido de que cree que el evaluador tiene la autoridad para juzgar un mérito del programa debido a la comprensión experta del área temática (es decir, la educación, la salud pública), y no a su experiencia y pericia como evaluador. Dado que Eisner utiliza la observación intensa (que incluye tanto la observación cuantitativa como la cualitativa, pero hace hincapié en esta última), se le coloca en un retoño que se inclina hacia el brazo de influencia constructivista en la rama de la valoración. Wolf y Owens (Owens, 1973; Wolf, 1979) se colocan en el brazo de influencia pospositivista porque sus perspectivas adhieren al principio de que los evaluadores deben buscar la verdad.

Hemos trasladado a Guba y Lincoln (2004) a la base del brazo de influencia constructivista para reflejar la ontología y la epistemología de su perspectiva, que se adhiere estrechamente a los principios básicos del paradigma constructivo. Su modelo teórico sirve como base para una serie de modelos que están influenciados por los principios del paradigma constructivista, tres de los cuales se han añadido al árbol de la teoría. Primero, el modelo de evaluación democrática deliberativa de House y Howe (1999) se 
ha incluido como su propio modelo separado del trabajo de justicia social de House, que ya está representado en el árbol. La evaluación democrática deliberativa se basa en los ideales de la labor anterior de House sobre la justicia social en la evaluación y su trabajo ha sido influenciado por los procesos presentados en Guba y el texto de Lincoln Fourth Generation Evaluation (Evaluación de cuarta generación). Así, la evaluación democrática deliberativa se ha colocado en el árbol encima de Guba y Lincoln.

House y Howe (1999, p. 102) creen que los evaluadores «deberían aceptar la autoridad, pero no el poder». Un evaluador que responde a los que tienen poder perpetúa la desigualdad, razón por la cual la inclusión es el primer criterio de evaluación democrática deliberativa. La inclusión evita el sesgo hacia alguna de las partes interesadas e involucra a todas las partes, con y sin poder, para participar en la evaluación. El diálogo es el siguiente criterio. El diálogo es necesario para asegurar que las contribuciones de los interesados estén bien pensadas y se puedan sopesar honestamente junto con todas las contribuciones a la evaluación de los demás interesados. La deliberación, criterio final para la evaluación democrática deliberativa, es la ponderación de cada contribución para generar una conclusión precisa. El enfoque de House y Howe se basa en valores. El hecho y el valor no son mutuamente excluyentes. Ambos conforman un continuo donde existe un término medio entre hechos brutos y valores básicos (House y Howe, 1999, p. 6). House y Howe describen el proceso de la evaluación democrática deliberativa de la siguiente manera: «podemos imaginarnos moviéndonos a lo largo del continuo valor-hecho desde las declaraciones de preferencias y valores recopilados a través del diálogo inicial, pasando por las deliberaciones basadas en principios democráticos, hasta declaraciones evaluativas de hechos» (House y Howe, 1999, p. 100).

El enfoque de Jennifer Greene también se ha agregado al árbol, directamente encima de House y Howe. El enfoque de compromiso con los valores de Greene (Greene, 2005) tiene sus raíces en los principios y procedimientos de la evaluación democrática deliberativa; aunque pone un énfasis adicional en los valores de las partes interesadas para enmarcar la evaluación. El enfoque de compromiso con los valores de Greene utiliza tres criterios de la evaluación democrática deliberativa: inclusión, diálogo y deliberación. Sin embargo, hace hincapié en la participación de las partes interesadas, lo que se asemeja mucho a los enfoques de evaluación participativa. También enfatiza explícitamente el uso de diseños de métodos mixtos y trabajo de campo dentro de sus evaluaciones.

El enfoque inclusivo de Donna Mertens (1999) podría considerarse un antecedente directo de Guba y Lincoln, pero es único en su énfasis en la diversidad y la inclusión de grupos diversos. Mertens es mejor conocida por su modelo de evaluación inclusiva/transformadora en el que el papel principal del evaluador es incluir a los grupos marginados, no actuar como tomadores de decisiones. El evaluador aboga por la inclusión de los grupos marginados, no por los grupos marginados. Mertens sostiene que los 
evaluadores que trabajan en un marco inclusivo deben hacerse las siguientes preguntas durante las etapas de planificación de la evaluación:

- ¿estamos incluyendo personas de ambos géneros, con capacidades diversas, así como de diferentes clases, culturas, edades, etnias, familias, ingresos, idiomas, ubicaciones, razas y sexualidades?,

- ¿qué barreras estamos levantando para excluir a una diversidad de personas?, y

— ¿hemos elegido las estrategias de recopilación de datos adecuadas, incluida la provisión de modos de comunicación privilegiados, para los diversos grupos? (Mertens, 1999, p. 8).

El objetivo de la inclusión es garantizar que la evaluación sea realizada en el contexto de la totalidad del programa de una manera que desaliente el sesgo; no es abogar por un grupo sobre otro. El objetivo principal de la evaluación inclusiva/transformadora es desafiar el statu quo en una búsqueda para transformar la sociedad. Mertens está de acuerdo y cita a Chelimsky, quien escribió que desafiar el statu quo es «nuestra tarea más importante y la mejor justificación de nuestro trabajo» (citado en Mertens, 1999 , p. 2). En este paradigma, las evaluaciones son una herramienta para combatir la desigualdad y la injusticia social.

\section{Resumen}

Y así, la discusión anterior del árbol teórico modificado representa nuestro pensamiento actual sobre cómo clasificar a los teóricos de la evaluación. Esta imagen actual de las teorías de evaluación prescriptiva guiará nuestro pensamiento sobre los problemas de evaluación hasta que futuros cambios se hagan necesarios.

\section{Referencias}

Donaldson, S. I., Christie, C. A., \& Mark, M. (Eds.). (2008). What Counts as Credible Evidence in Evaluation and Evidence-based Practice? Thousand Oaks, CA: Sage.

Eisner, E. (2004). The roots of connoisseurship and criticism: A personal journey. En M. Alkin (ed.), Evaluation roots: Tracing theorists' views and influences. Thousand Oaks, CA: Sage.

Greene, J. C. (2005). A value-engaged approach for evaluating the Bunche-Da Vinci learning academy. New Directions for Evaluation, 106, 27-47. 
Guba, E., y Lincoln, Y. (2004). The roots of fourth generation evaluation: Theoretical and methodological origins. En M. Alkin (ed.), Evaluation roots: Tracing theorists' views and influences. Thousand Oaks, CA: Sage.

Henry, G., Julnes, G. J., y Mark, M. (1998). Realist evaluation. San Francisco: Jossey-Bass.

House, E., y Howe, K. (1999). Values in evaluation and social research. Thousand Oaks, CA: Sage.

Mark, M., Henry, G., y Julnes, G. (2000). Evaluation: An integrated framework for understanding, guiding, and improving policies and programs. San Francisco, CA: JosseyBass.

Mathison, S. (ed.). (2005). Encyclopedia of evaluation. Thousand Oaks, CA: Sage.

Mertens, D. (1999). Inclusive evaluation: Implications of transformative theory for evaluation. American Journal of Evaluation, 20(1), 1-14.

Owens, T. (1973). Education evaluation by adversary proceeding. En E. R. House (ed.), School evaluation: The politics and process. Berkeley, CA: McCutcheon.

Scriven, M. (2003). Evaluation in the new millennium: The transdisciplinary view. En S. I. Donaldson y M. Scriven (eds.), Evaluating Social Programs and Problems: Vision for the New Millennium. Mahwah, NJ: Erlbaum. pp. 19-42.

Stake, R. (2004). Stake and responsive evaluation. En M. Alkin (ed.), Evaluation roots: Tracing theorists' views and influences. Thousand Oaks, CA: Sage.

Wholey, J. S. (2004). Using evaluation to improve performance and support policy decision making. En M. Alkin (ed.), Evaluation roots: Tracing theorists' views and influences Thousand Oaks, CA: Sage.

Wolf, R. L. (1979). The use of judicial evaluation methods in the formulation of educational policy. Educational Evaluation and Policy Analysis, 1(93), 19-28. 